\title{
Endogenous cannabinoids trigger the depolarization-induced suppression of excitation in the lateral amygdala
}

\author{
Sodikdjon A. Kodirov, ${ }^{1,2}$ Julia Jasiewicz, Parisa Amirmahani, Dimitrios Psyrakis, \\ Kathrin Bonni, Michael Wehrmeister, and Beat Lutz \\ Institute of Physiological Chemistry and Pathobiochemistry, University Medical Center Mainz, 55099 Mainz, Germany
}

\begin{abstract}
The amygdala is a key area of the brain where the emotional memories are stored throughout the lifespan. It is well established that synapses in the lateral nucleus of amygdala (LA) can undergo long-term potentiation, a putative cellular correlate of learning and memory. However, a type of short-term synaptic plasticity, known as depolarization-induced suppression of excitation (DSE), has not been studied previously in the amygdala in general and in the LA in particular. In this study we aimed to prove either the absence or the presence of this phenomenon in the LA. Our data demonstrate for the first time that DSE is present in the LA and that it modulates the cortical excitatory synaptic input into this region. The existence of this type of retrograde neurotransmission in glutamatergic pyramidal neurons of the LA suggests that the axonal terminals of cortical inputs do possess functional type 1 cannabinoid receptors, and provides a novel insight regarding inputs into the LA. Further experiments indeed revealed endocannabinoids as the messenger for this retrograde signaling in the LA. In conclusion, the DSE may play a functional role in synaptic plasticity and related emotional memory processing in the LA.
\end{abstract}

Pavlovian classical conditioning (Pavlov 1927) is accompanied by an increase in synaptic strength within the fear circuit of the amygdala (McKernan and Shinnick-Gallagher 1997; Nader et al. 2000). The involvement of the amygdala in different forms of behavior is a long-standing research interest (Morgane and Kosman 1957; Gallagher et al. 1977). The lateral amygdala (LA) is modulated by excitatory afferents and involved in synaptic plasticity, i.e., the counterpart that underlies learning and memory at neuronal level. The memory formation in the LA can easily be demonstrated since synapses in this region can undergo long-term potentiation (LTP). Long-term synaptic plasticity has been extensively explored and its properties in the amygdala are well established (McKernan and Shinnick-Gallagher 1997; Mahanty and Sah 1998; Royer and Pare 2003; Kodirov et al. 2006). In the amygdala, LTP induction can be either $N$-methyl-D-aspartate (NMDA) glutamate receptor dependent or independent (Gewirtz and Davis 1997; Mahanty and Sah 1998).

Several brain areas and their neurons are also prone to shortterm changes at the synaptic level (Diana and Marty 2004). However, a type of short-term synaptic plasticity, known as depolarization-induced suppression of excitation (DSE), has not been studied previously in the amygdala. DSE, similar to depolarization-induced suppression of inhibition (DSI), recruits a cross talk between pre- and postsynaptic neurons. DSI has been extensively studied in hippocampus (Pitler and Alger 1994; Chevaleyre et al. 2007), cerebellum (Vincent et al. 1992; Kreitzer and Regehr 2001; Duguid and Smart 2004), and cerebral cortex (Fortin and Levine 2007); however, there are fewer studies dedicated to DSE (Brown et al. 2003; Straiker and Mackie 2005;

1Present address: University of Texas at Brownsville, Department of Biological Sciences, Center for Biomedical Studies, 80 Fort Brown, LHSB 2.816, Brownsville, TX 78520, USA.

${ }^{2}$ Corresponding author.

E-mail Sodikdjon.Kodirov@utb.edu; fax (956) 882-5043.

Article is online at http://www.learnmem.org/cgi/doi/10.1101//m.1663410.
Uchigashima et al. 2007). The suppression can be achieved not only by depolarization of the postsynaptic neuron, but also by brief trains of action potentials (APs) evoked by stimulation of innervating fibers (Brown et al. 2003). This phenomenon is known as synaptically evoked suppression of excitation (SSE), which involves the mGluR1-mediated release of endocannabinoids. DSI has also been observed between two synaptically connected cholecystokinin-positive and parvalbumin-negative interneurons in stratum radiatum of the hippocampus (Ali 2007) and neurons from the latter structure in culture (Hashimotodani et al. 2007). A similar phenomenon for the inhibitory neurotransmission using the postsynaptic neuron/synaptic bouton preparation from the basolateral amygdala (BLA) in culture and brain slices has been shown (Zhu and Lovinger 2005). The signaling cascade under these conditions is initiated in the postsynaptic neuron and, therefore, is regarded as a retrograde neurotransmission. The latter neurotransmission occurs as a result of transient release of endogenous cannabinoids. Also the involvement of arachidonic acid as a retrograde messenger in the long-term plasticity of the hippocampus has been postulated and subsequently revealed both in vivo and in vitro (Williams et al. 1989).

Our results demonstrate that DSE is present in a majority of LA pyramidal neurons. Further experiments reveal endogenous cannabinoids as the messengers for this retrograde signaling in the LA. The role of endocannabinoids for brain function is essential as ongoing research in this area reveals (Stella et al. 1997; Wilson et al. 2001; Marsicano et al. 2002; Tzounopoulos et al. 2007). Recent investigations show that endocannabinoids disrupt the vital functions of the responsible brain areas, i.e., learning and memory (Hohmann et al. 2005; Chevaleyre et al. 2007).

\section{Results}

We characterized the depolarization-induced suppression of excitation (DSE) in pyramidal neurons of the LA. These cells were distinguished by frequency adaptation properties (Fig. 1C) of their 

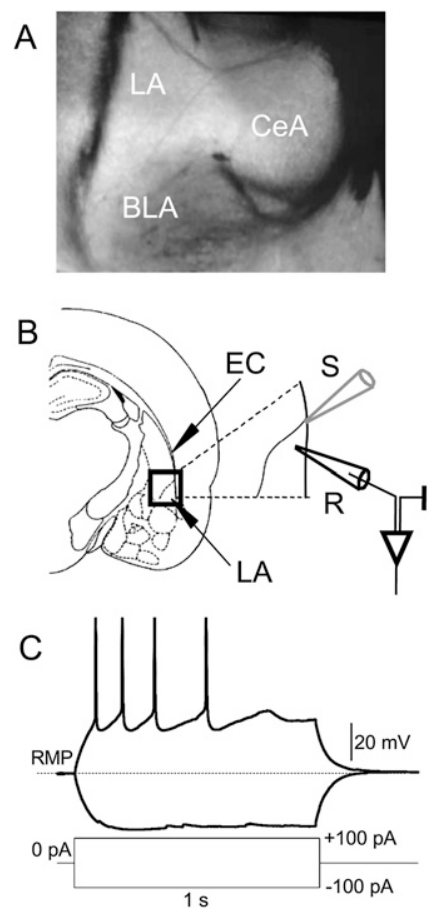

Figure 1. LA brain slice and action potential (AP) waveform of pyramidal neuron. $(A)$ Brain slice containing the amygdala with its major nuclei: LA, lateral amygdala; BLA, basolateral amygdala; $\mathrm{CeA}$, central amygdaloidal nucleus. (B) Schematic representation of coronal slice and experimental conditions; $E C$, external capsule; R, recording electrode; $S$, stimulating electrode. (C) Typical property of pyramidal neuron; RMP, resting membrane potential $(-55.8 \mathrm{mV})$. Scale bars are shown.

APs. The APs were evoked from the resting membrane potential (RMP) by injection of constant depolarizing currents (100 pA, 1-s duration; Fig. 1C). The mean value of RMP in recorded pyramidal neurons was $-55.2 \pm 2.1 \mathrm{mV}(n=35)$. Note that APs were elicited consistently at the end of DSE experiments in order to not interfere with the effects of the depolarizations (up to $+40 \mathrm{mV}$ ) occurring during neuronal firing, since the depolarization of cell membrane potential can trigger AP generation, the elevation of intracellular $\mathrm{Ca}^{2+}$, LTP (if paired), long-term depression, and possibly more.

\section{Depolarization-induced suppression of spontaneous EPSCs}

In order to elucidate DSE in pyramidal cells (see Fig. 1C) of LA, spontaneous excitatory postsynaptic currents (sEPSCs) were recorded at a holding potential of $-70 \mathrm{mV}$ (Fig. 2A,B). The inhibitory neurotransmission mediated by $\gamma$-aminobutyric acid receptor type $\mathrm{A}\left(\mathrm{GABA}_{\mathrm{A}}\right)$ in this, and subsequent experiments, was blocked by application of $100 \mu \mathrm{M}$ picrotoxin (PTX). The average values of current frequencies and amplitudes under baseline conditions in pyramidal cells were $7.0 \pm 1.8 \mathrm{~Hz}$ and $8.0 \pm 1.2 \mathrm{pA}(n=10)$, respectively (Fig. 2F,G). The 10-s depolarization to $0 \mathrm{mV}$ significantly decreased the frequency of sEPSCs to $5.5 \pm 1.4 \mathrm{~Hz}(n=10$, $P<0.01)$. Note that a comparable retrograde neurotransmission in glutamatergic pyramidal neurons of the LA was observed also by 5-s depolarizations (data not shown). The amplitude distributions of sEPSCs during baseline (black bars) and after the depolarization (open) are shown in Figure 2E. The sEPSCs' amplitudes remained unchanged and the corresponding mean value was $7.9 \pm 1.2 \mathrm{pA}$ ( $n=10, P=0.5$ ). Further, the cumulative probability histograms consistently revealed identical results, i.e., the modulation of presynaptic release (frequency, $P<0.02$, Kolmogorov-Smirnov test; Fig. 2C) of glutamate by DSE without affecting the properties of postsynaptic receptors (amplitude; Fig. 2D). Our results, for the first time, reveal DSE occurrence in the LA and the underlying mechanism is a modulation of presynaptic neurotransmitter release (since only the frequency was affected; see Fig. 2) caused by the depolarization of the postsynaptic neuron. Note that a complete inhibition, lasting a few seconds, with a gradual recovering rate was not always observed in this (LA) preparation under these physiological conditions, i.e., in the absence of either carbachol or a higher concentration of $\mathrm{K}^{+}$, which are used in order to enhance the frequency of neurotransmitter release. In six out of 16 cells, none of the parameters of sEPSCs were significantly affected under identical conditions by a brief depolarization $(10.0 \pm 3.3$ vs. $9.5 \pm 2.9 \mathrm{~Hz}, n=6, P=0.3)$.

\section{Depolarization does not affect the miniature EPSCs}

DSE of sEPSCs (Fig. 2) compromises the glutamate release derived both by AP-dependent and -independent pathways. In order to

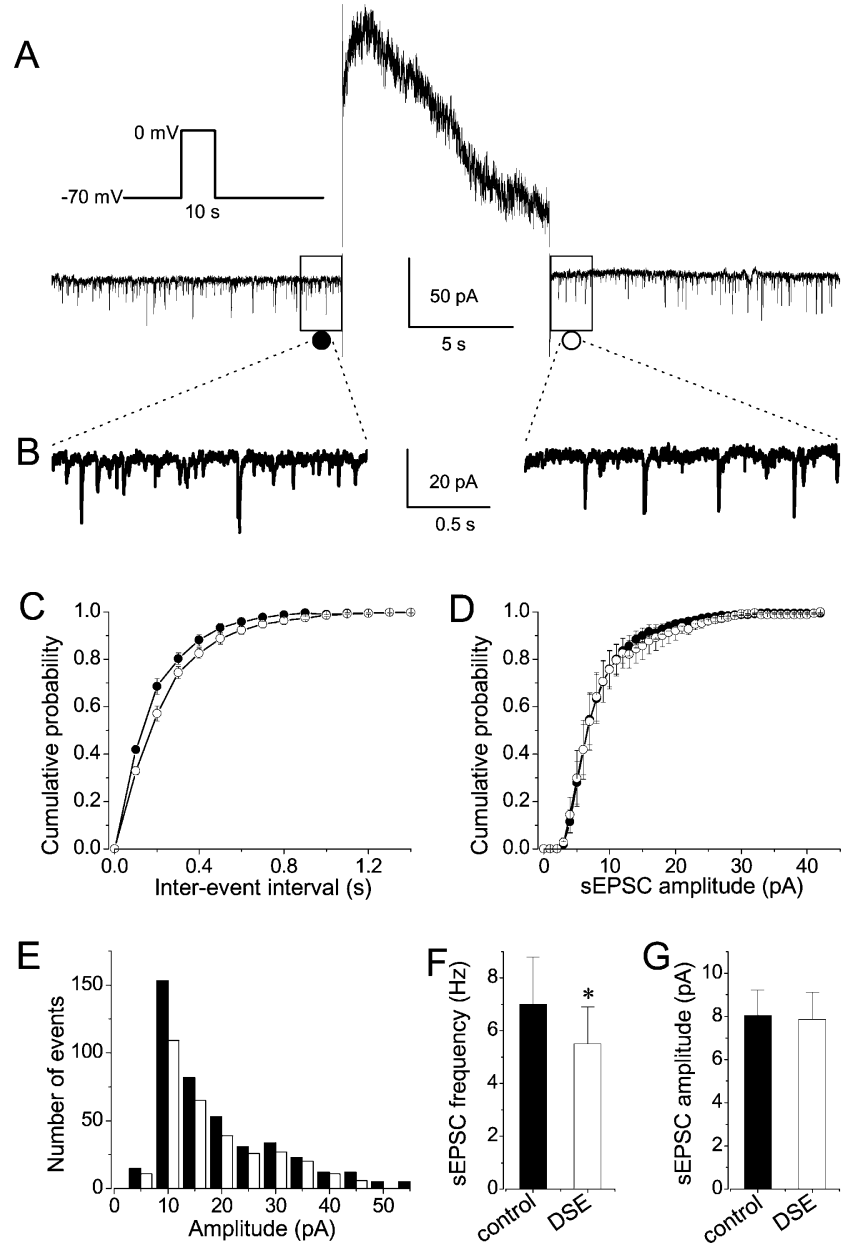

Figure 2. Depolarization-induced suppression of spontaneous EPSCs in pyramidal neurons of LA. (A) Spontaneous EPSCS recorded in the presence of $100 \mu \mathrm{M}$ PTX at a holding potential of $-70 \mathrm{mV}$. (B) Spontaneous EPSCs pattern immediately before and after the 10-s depolarizing step to $0 \mathrm{mV}$ in an expanded timescale. Scale bars are shown. $(C, D)$ Cumulative frequency $(P<0.02$, Kolmogorov-Smirnov test) and amplitude $(P=0.5)$ probabilities, respectively. Black and open symbols are SEPSCs amplitude/rate before and after the DSE induction, respectively. ( $E$ ) Amplitude distributions of sEPSCs (5 pA equal bins). Note significant decrease in number of events (317) during the DSE compared to the baseline (427). $(F, G)$ Mean frequency $\left(n=10,{ }^{*} P<0.01\right.$, paired $t$-test) and amplitude values of sEPSCs $(P=0.5)$. Error bars are \pm SEM. Statistical data represent mean values in 10 out of 16 cells tested. 
examine whether or not the glutamate release in the absence of APs (mEPSCs) is susceptible to DSE, the following experiments were conducted. First we obtained recordings under standard initial conditions (similar to Figs. 1C, 2A) then we applied either $100 \mathrm{nM}$ or $1 \mu \mathrm{M}$ tetrodotoxin (TTX) to the same neuron. In this set of experiments, either eEPSCs (data not shown) or the APs were continuously elicited until an actual effect of TTX was observed as presented in Figure 3A. Thus, no APs could be evoked in the presence of TTX and, subsequently, the AP triggered neurotransmissions were completely eliminated. Soon after this procedure the mEPSCs were recorded in the presence of both PTX and TTX at a holding potential of $-70 \mathrm{mV}$ (Fig. 3B). When the stable baseline (black symbols/bars) frequency of mEPSCs was at least $2 \mathrm{~Hz}$, the membrane potential was changed to $0 \mathrm{mV}$ for $10 \mathrm{~s}$ and recording was continued immediately upon termination of the step (open). This depolarizing step did not significantly alter the appearance of the mEPSCs judged visually from the recording in an expanded timescale (Fig. 3C). The cumulative frequency (Fig. 3D) and amplitude (Fig. 3E) probabilities revealed the same conclusion. Further-
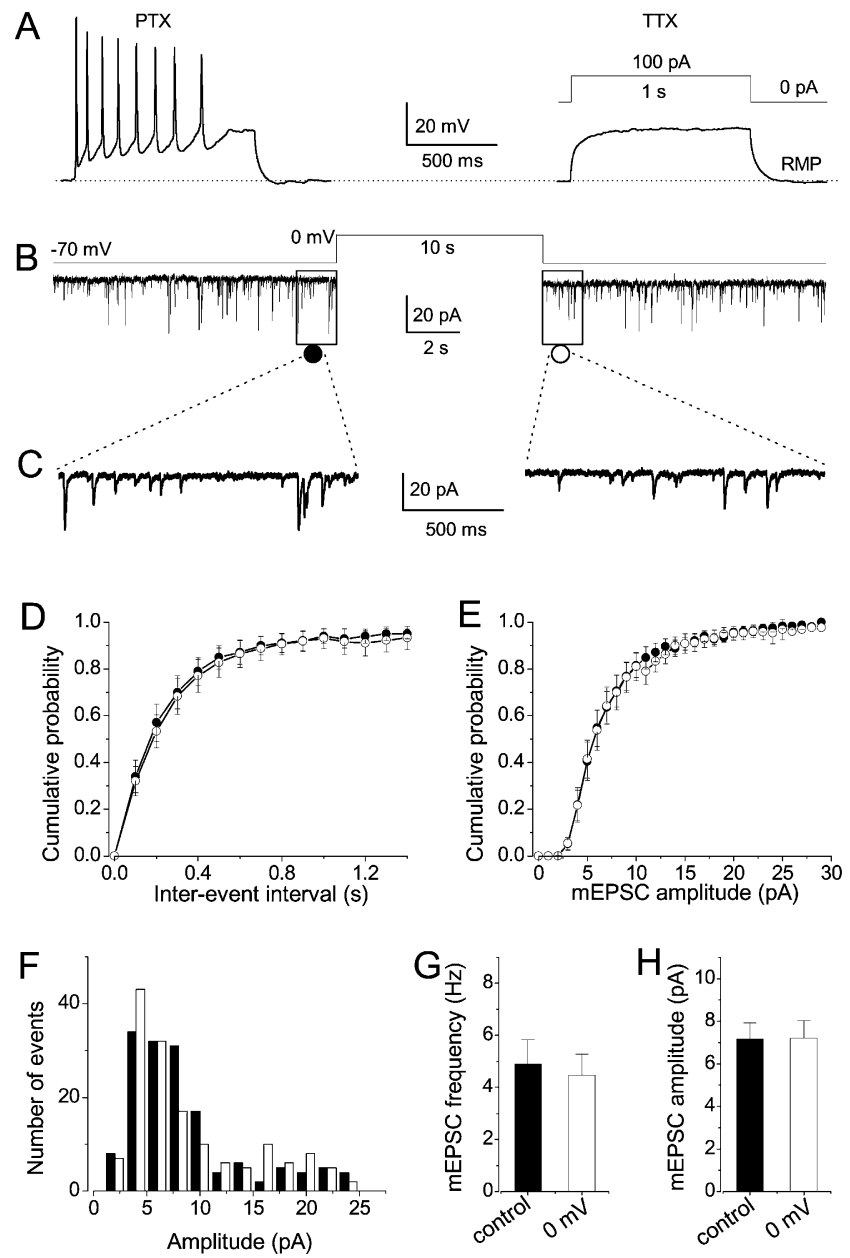

Figure 3. Miniature EPSCs are not susceptible to DSE. $(A)$ Evoked APs in presence of PTX and their complete elimination after the application of TTX. (B) mEPSCs recorded in the presence of both PTX and TTX at a holding potential of $-70 \mathrm{mV}$. (C) Recorded mEPSCs before and after the 10-s depolarizing step to $0 \mathrm{mV}$ in an expanded time frame. Scale bars are shown. $(D)$ Cumulative frequency and $(E)$ amplitude probabilities. Black and open symbols are mEPSCs amplitude/rate before and after the DSE induction, respectively. $(F)$ Number and amplitude distributions of mEPSCs during the baseline (156) and after the depolarization (151). $(G, H)$ Mean frequency and amplitude values of mEPSCs $(n=9, P>0.05)$. Error bars are \pm SEM. more, no alterations in the mEPSCs number distributions during the baseline (156) and after the depolarization (151) were observed (Fig. 3F). Finally, the average frequency and amplitude magnitude of mEPSCs $(n=9, P>0.05)$ remained almost the same under two conditions (Fig. 3G,H). Thus, similar to previous observations in the cornu ammonis (CA1) area of the hippocampus (Alger et al. 1996), the mEPSCs in pyramidal neurons of the LA were not vulnerable to DSE.

\section{Depolarization-induced suppression of evoked EPSCs}

Since the amygdala is believed to be a neuronal circuit of emotional memories, we next performed experiments by exciting the ionotropic glutamate receptors via cortical inputs. The excitatory postsynaptic currents in the LA were evoked (eEPSCs) by the stimulation of the external capsule (Fig. 1B). The baseline eEPSCs' magnitude during $42 \mathrm{~s}$ was recorded at a holding potential of $-70 \mathrm{mV}$ and a single 10-s voltage step of $0 \mathrm{mV}$ was applied (Fig. $4 \mathrm{~A}-\mathrm{C}$ ). This relatively brief depolarization (Chen et al. 2003) significantly decreased the amplitude of eEPSCs recorded immediately upon repolarization in 14 out of 21 pyramidal neurons tested $(83.2 \pm 2.1 \%$ of baseline, $n=14, P<0.001$; Fig. $4 \mathrm{~F})$. The magnitude of DSE was relatively small; it reached the steady-state level gradually and only a slow recovery was observed during the tested range (at least $80 \mathrm{~s}$; Fig. 4B,C). Thus, DSE in the LA during this time frame in the majority of experiments was not a strictly transient phenomenon compared to that in the cerebellum (Kreitzer and Regehr 2001) and hippocampus (SA Kodirov, K Bonni, and D Psyrakis, unpubl.). Nevertheless, in several cells we have observed a reversible suppression induced by a short depolarizing step (see Fig. 4B). The magnitude of eEPSCs in a subpopulation of pyramidal neurons remained unchanged after the depolarization with a mean value of $101.2 \pm 1.8 \%$ compared to the baseline ( $n=7, P=0.4$; data not shown).

In additional experiments, we used the $\mathrm{Cs}^{+}$-based intracellular solution to inhibit the outward $\mathrm{K}^{+}$currents activated upon depolarization of postsynaptic neurons and subsequently to achieve more adequate voltage changes toward the distal dendrites. The maximal magnitude of DSE under these conditions had a mean value of $82.2 \pm 5.5 \%$ of baseline ( $n=4, P<0.05$; data not shown), which is comparable with those obtained using the intracellular solution containing $\mathrm{K}^{+}$-gluconate (see Fig. 4). Note that the pairing paradigm is also employed with the latter solution in the recording electrode, which enables optimal conditions for the induction of LTP (Kodirov et al. 2006). Therefore, we proceeded with the entire study using $\mathrm{K}^{+}$-gluconate-based solution (Chen et al. 2003), which also enabled the identification of pyramidal neurons in the LA.

\section{Retrograde modulation of excitatory neurotransmission via $\mathrm{CB}_{1} \mathrm{Rs}$}

The DSI in many brain areas occurs during the release of endogenous cannabinoids and thus the latter could be of functional essentiality. This particular retrograde signaling messenger similar to DSI might be involved in DSE observed in LA. Therefore, we used a selective antagonist of type 1 cannabinoid receptors $\left(\mathrm{CB}_{1} \mathrm{Rs}\right) \mathrm{AM}-251$. First, we studied the effects of this antagonist on evoked EPSCs at $0.05 \mathrm{~Hz}$ in pyramidal cells. The amplitude of eEPSCs remained unchanged during at least $10 \mathrm{~min}$ after the application of $10 \mu \mathrm{M}$ AM-251 $(100.3 \pm 2.4 \%$ of baseline, $n=8, P=$ 0.9; Fig. 5A,B). There are two possible explanations for this observation: First, there might be insufficient or no tonic release of endogenous cannabinoids and subsequent activation of $\mathrm{CB}_{1} \mathrm{Rs}$ in the LA. The latter explanation has also been applied to the steady-state level of endocannabinoid ligands involved in retrograde GABAergic neurotransmission in the CA1 area of the hippocampus (Chen et al. 2003). Second, the concentration of the antagonist perhaps was not sufficient to affect the basal excitatory 

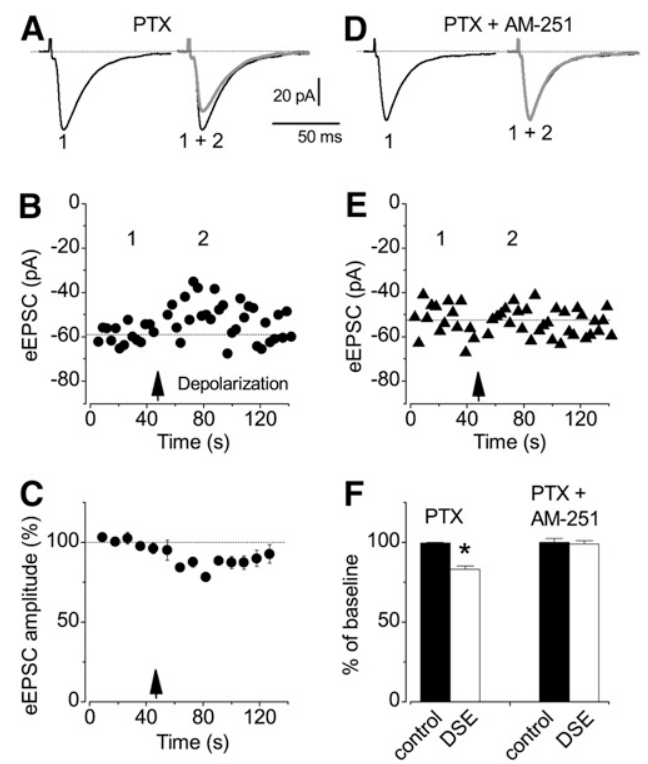

Figure 4. Depolarization-induced suppression at excitatory synapses between cortical inputs and pyramidal neurons. $(A)$ The magnitude of eEPSCs before (left) and after the application of voltage step to $\pm 0 \mathrm{mV}$ (right), are superimposed. (B) Data points collected at $0.33 \mathrm{~Hz}$ from the same single representative experiment shown in $A$. (C) Normalized mean amplitude values $(n=14)$. ( $D, E)$ Recordings in the presence of $10 \mu \mathrm{M} \mathrm{AM}$ 251. $(F)$ DSE magnitude before $(n=14, * P<0.01)$ and after the application of AM-251 $(n=9)$. The sample traces in $A$ and $D$ are the average of EPSCs obtained at indicated time frames within $42 \mathrm{~s}$. Scale bars for $A$ and $D$ are identical. Error bars are \pm SEM.

neurotransmission. This $\mathrm{CB}_{1}$ receptor antagonist was applied only if a significant DSE was present under control conditions. Thus, the DSE in the presence of $10 \mu \mathrm{M}$ AM-251 (Fig. 4D,E) was significantly diminished $(97.2 \pm 1.4 \%$ of baseline, $n=9, P=0.2$; Fig. $4 \mathrm{~F}$ ). The passive membrane properties of the two abovementioned subpopulations of pyramidal neurons were not significantly different. We observed only significant differences in active properties of neurons manifested by analyzing the number of spikes triggered during $1 \mathrm{~s}$ depolarization (enabled by $100 \mathrm{pA}$ ) in DSE susceptible and nonsusceptible cells $(7.9 \pm 0.8, n=14$ vs. $3.6 \pm 1.2$ spikes/s, $n=7$, respectively; $P<0.005$ ).

\section{DSE and the role of intracellular $\mathrm{Ca}^{2+}$}

Next, our data revealed that retrograde neurotransmission in the LA is initiated by a transient increase of $\mathrm{Ca}^{2+}$ in postsynaptic neurons, which occurs also during the course of AP generation under physiological conditions. The crucial role of intracellular $\mathrm{Ca}^{2+}$ for the DSE was evaluated using the EGTA. The eEPSCs were recorded with a similar $\mathrm{K}^{+}$-gluconate-based intracellular solution as in previous experiments (see Fig. 4), but the content of EGTA was increased from 0.2 to $10 \mathrm{mM}$. Since we used the low-resistance electrodes, the equilibration time prior to recordings was consistently at least $5 \mathrm{~min}$. The superimposed average magnitude of evoked EPSCs, before (Fig. 6A, left panel) and after the depolarizing step of $70 \mathrm{mV}$ (Fig. 6A, right panel), revealed no significant suppression in pyramidal cells. Figure $6 \mathrm{~B}$ represents the amplitude data points obtained from the same recording. Thus, under these conditions the responses of pyramidal neurons were rather homogeneous ( $99 \pm 2.3 \%$ of baseline, $n=14, P=0.8$; Fig. $6 \mathrm{C}$ ). These results reveal that during the DSE inducing paradigm, a sufficient voltage-dependent $\mathrm{Ca}^{2+}$ influx was achieved; rise in intracellular $\mathrm{Ca}^{2+}$ concentration serves as a key component leading to endogenous cannabinoid ligands release (see Fig. 8, below).

\section{Depolarization-induced increase in paired-pulse ratio}

Any observable changes in paired-pulse ratio (PPR) hint to the presynaptic effects of given conditions; a constant ratio will predict the postsynaptic origin of actual effects. Therefore, we aimed to elucidate whether or not the DSE of evoked EPSCs involves either the probability of glutamate release or postsynaptic receptor rearrangements. Experiments were conducted under similar conditions as presented in Figure 4A,B except for the stimulation paradigm. The latter consisted of two pulses with an interstimulus interval of 30, 50, and $55 \mathrm{~ms}$. After the baseline recording period the membrane potential was subjected to either 1 or $10 \mathrm{~s}$ depolarization. The eEPSCs waveforms before and after the depolarization to $\pm 0 \mathrm{mV}$ are shown in Figure 7A (black and gray traces, respectively). The collected (every $3 \mathrm{~s}$ ) data reveal a significant decrease in the amplitude of the first EPSCs (Fig. 7B) evoked immediately after the depolarization; black and gray lines correspond to the average traces shown in Figure 7A. Note relatively unstable baseline amplitudes of first EPSCs; at least one of the data points is out of range. However, all data points were included both in average traces and mean data. The amplitude of the second EPSCs was not significantly affected under these conditions, although a slight tendency toward suppression was observed. This consistent DSE correlated with the increased paired-pulse ratio in the same pyramidal neuron (Fig. 7C) and six other cells (Fig. 7D; $137.8 \pm 15.2 \%$ of baseline, $\left.n=7,{ }^{*} P<0.05\right)$. Our experiments reveal that these effects were to some extent reversible within up to $2 \mathrm{~min}$ (106.7 $\pm 11.0 \%$ of baseline, $P>0.05$ ). Thus, a consistent conclusion, in regard to the presynaptic site of final effects of depolarization (DSE) on spontaneous events, could also be extended to those of evoked EPSC.

\section{Further support for the modulation of presynaptic neurotransmitter release by DSE}

Our results obtained by examining the frequency of spontaneous EPSCs revealed that only the glutamate release was targeted by DSE (Fig. 2C). Next, the examination of EPSCs evoked using a paired-pulse paradigm led to the same consistent results (Fig. 7). We have performed additional analyses using a previously described paradigm (Faber and Korn 1991; Alger et al. 1996). For this purpose we have used original experiments similar to those illustrated in Figure 4A-C. The magnitude of DSE and the coefficient of variation $\left(\mathrm{CV}^{2}\right)$ ratio in the same individual pyramidal neurons (Fig. 8A, $n=14$ ) was estimated and subsequently plotted.
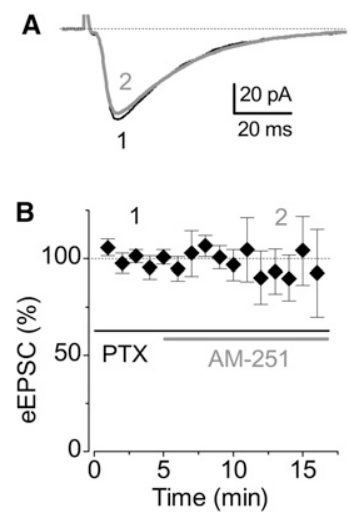

Figure 5. Insufficient tonic release of endogenous cannabinoids in the LA. $(A)$ The $C B_{1} R$ antagonist did not affect the basal amplitude of evoked EPSCs. (B) Summary data $(100.3 \pm 2.4 \%$ of baseline, $n=8, P=0.9)$. The sample traces are the average of EPSCs obtained at indicated time frames within $5 \mathrm{~min}$. Scale bars are shown and error bars are \pm SEM. 

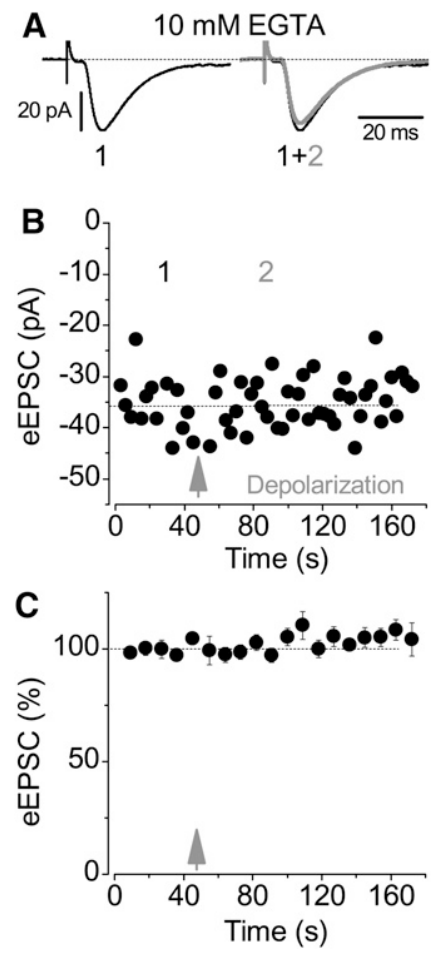

Figure 6. DSE in the LA is $\mathrm{Ca}^{2+}$ influx dependent. (A) Average magnitude of evoked EPSCs before (left) and after the depolarization $( \pm 0 \mathrm{mV}$, right); identical scale bars for representative traces are shown. The eEPSCs were recorded using the $\mathrm{K}^{+}$-gluconate-based intracellular solution containing $10 \mathrm{mM}$ EGTA at a holding potential of $-70 \mathrm{mV}$. (B) Data points reflecting the amplitude of eEPSCs during the entire course of experimental paradigm. (C) Normalized mean amplitude values in pyramidal cells $(n=14)$. Error bars are \pm SEM.

In the majority of pyramidal cells (11 out of 14$)$, the DSE in individual cells (abscissa axis) correlated with a decrease in $\mathrm{CV}^{2}$ ratio; only in three pyramidal cells did we observe an increase in $\mathrm{CV}^{2}$ ratio (ordinate). The average magnitude of $\mathrm{CV}^{2}$ ratio in recorded neurons was $83.5 \pm 7.6 \%$. The latter value was closely situated on the diagonal line separating the pure presynaptic site (Pre) from the mixed pre- and postsynaptic one (Pre/ Post). Nevertheless, since the $\mathrm{CV}^{2}$ ratio did not remain constant (either decreased or increased) after the depolarizing protocol, we conclude that the presynaptic site is involved in DSE (Fig. 8A).

DSE in the LA occurs as follows: The AP generation in the postsynaptic cell leads to elevated intracellular $\mathrm{Ca}^{2+}$ concentration that subsequently results in the release of endogenous cannabinoids. These in turn activate $\mathrm{CB}_{1} \mathrm{Rs}$ in the presynaptic terminal, which finally decreases in a retrograde fashion (Fig. 8C) the glutamate mediated anterograde neurotransmission (Fig. 8B).

\section{Discussion}

Depolarization of postsynaptic cells with a voltage step of moderate amplitude resulted in suppression of both spontaneous and evoked glutamatergic neuro- transmissions in the majority of pyramidal neurons studied in the LA. This is a form of short-term synaptic plasticity known as depolarization-induced suppression of excitation in different areas of the brain.

The pyramidal neurons of the lateral amygdala did not exhibit a homogenous DSE of evoked EPSCs and we sometimes observed a delay in DSE onset, which to some extent is similar to those of DSI in cultured hippocampal neurons (Hashimotodani et al. 2007). Unlike in the cerebellum (Kreitzer and Regehr 2001), hippocampus (Chen et al. 2003), striatum (Uchigashima et al. 2007), and dorsal cochlear nucleus (Tzounopoulos et al. 2007), the DSE in several pyramidal neurons of the LA was not a strictly transient phenomenon. Although, in some neurons we observed a transient DSE during the course of the experiments (see Fig. 4B). The maximal magnitude of DSE under our experimental conditions was comparable with those seen in pyramidal neurons of the CA1 area of the hippocampus (Chen et al. 2003). Likewise in the latter preparation (Alger et al. 1996), the DSE in pyramidal neurons of the LA was attributed to only AP triggered glutamate release, since the depolarization did not affect the properties of mEPSCs (Fig. 3).

Antagonizing the effects of endogenous cannabinoids (transiently released during depolarization) by selective $\mathrm{CB}_{1} \mathrm{Rs}$ blocker AM-251 reversed DSE in the LA. This antagonist did not significantly affect the basal excitatory neurotransmission into the pyramidal neurons (Fig. 5); consistent with the fact that DSE is an activity-dependent (in postsynaptic neuron) phenomenon and requires a transient release of endocannabinoids. These endocannabinoids comprise the two natural ligands, 2-AG (sn-2 arachidonylglycerol) and anandamide (Devane and Axelrod 1994), which bind to $\mathrm{CB}_{1}$ Rs.

It should be noted that the effects of $\mathrm{CB}_{1}$ Rs agonists and antagonists on basal neurotransmission are sometimes controversial depending on both the brain areas and the type of neurotransmitters studied. Often, the experiments are not straightforward; for example, the effect of antagonists is observed when the co-application approach was used in order to first activate $\mathrm{CB}_{1} \mathrm{Rs}$. Under these conditions, the exogenous agonist is not able to activate $\mathrm{CB}_{1} \mathrm{Rs}$ in the continuous presence of an antagonist (Takahashi and Linden 2000; Katona et al. 2001; Fortin and Levine 2007). Moreover, WIN 55212-2 decreases the amplitude of EPSCs
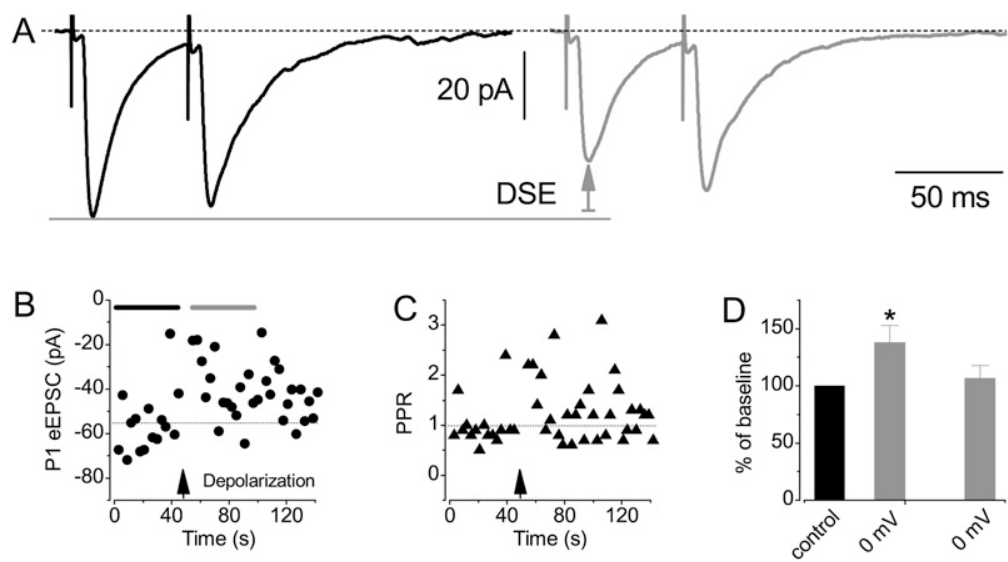

Figure 7. Depolarization-induced increase in paired-pulse ratio (PPR). ( $A$ ) EPSCs in pyramidal neurons were evoked at holding potential of $-70 \mathrm{mV}$ by the paired stimulation of the cortical inputs (black trace); interstimulus interval was kept at $55 \mathrm{~ms}$. Depolarization of membrane potential to $\pm 0 \mathrm{mV}$ significantly decreased the amplitude of EPSC (gray) evoked by the first pulse (P1; DSE). Traces are the average of 15 responses recorded every $3 \mathrm{~s}$ before and after depolarization, respectively. $(B, C)$ The amplitude of first EPSCs and the PPR during the entire course of the experiment shown in $A$. (D) Normalized values reveal a reversible increase in PPR $\left(n=7,{ }^{*} P<0.05\right)$; error bars are \pm SEM. The amplitude and timescale bars for the two panels in $A$ are identical. 


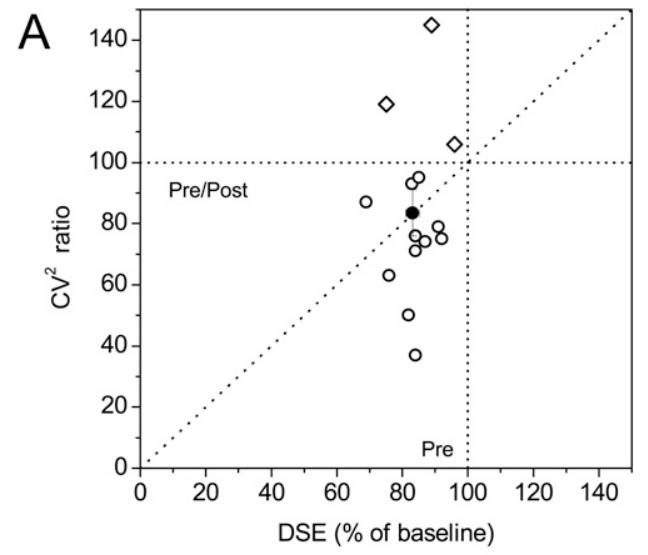

B
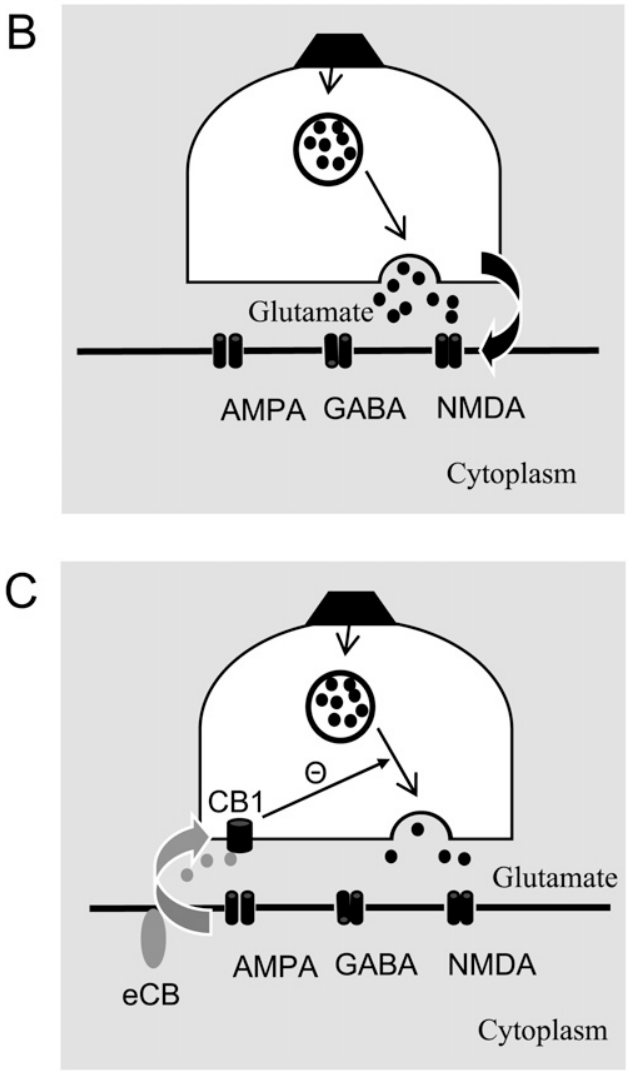

Figure 8. DSE in the LA mimics the decrease in glutamate release. $(A)$ Magnitude of DSE and ratio of the coefficient of variation $\left(\mathrm{CV}^{2}\right)$ in individual pyramidal cells $(n=14)$. The individual DSE and a decrease in $\mathrm{CV}^{2}$ ratio are shown $(O)$. Mean DSE and $\mathrm{CV}^{2}$ ratio are shown with a single symbol $(\bullet)$. Note an increase $(\diamond)$ in $\mathrm{CV}^{2}$ ratio in three pyramidal cells. Original data are shown in Figure 4. Both $x$ and $y$ error bars are \pm SEM. (B) The simplified anterograde neurotransmissions in the lateral amygdala caused by the AP triggered in the presynaptic site. (C) Endocannabinoid mediated retrograde transmission initiated by the AP in the postsynaptic pyramidal neuron; AMPA, $\alpha$-amino-3-hydroxy-5-methyl-4-isoxazolepropionic acid glutamate receptor; NMDA, $\mathrm{N}$-methyl-D-aspartate glutamate receptor; GABA, $\gamma$-aminobutyric acid receptor; $C B 1$, cannabinoid receptor type 1; eCB, endogenous cannabinoid ligands.

recorded from the L5 pyramidal neuron if evoked by the stimulation of the same layer, but not L2/3 (Fortin and Levine 2007); it affects neurotransmission in the BLA complex, but not in central and medial nuclei (Katona et al. 2001; Azad et al. 2003; Chevaleyre et al. 2007); AM-251 does not affect the baseline EPSPs' magnitude, but blocks long-term depression (LTD) and subsequently unmasks LTP (Tzounopoulos et al. 2007). Interestingly, the activation of $\mathrm{CB}_{1}$ Rs and subsequently the modulation of LTP by endocannabinoids was dependent on the induction paradigm (Slanina et al. 2005). The endocannabinoid 2-AG also has no significant effect on the EPSPs' amplitude. However, it blocks IPSCs (Hashimotodani et al. 2007) and prevents LTP induction in the CA1 area of the hippocampus (Stella et al. 1997).

The retrograde suppression of excitation in the LA was shown to be $\mathrm{Ca}^{2+}$ dependent (Fig. 6), since intracellular buffering with EGTA prevented the effects of short depolarization. Furthermore, two sets of experimental data proved that DSE of both the spontaneous (changes in frequency) and evoked EPSCs (increased PPR) was targeted to the presynaptic neurons/fibers. Finally, these results were consistent with the outcome of additional analyses synergistically concerning both the magnitude of DSE of evoked EPSCs and the coefficient of variation $\left(\mathrm{CV}^{2}\right)$ ratio in the same individual pyramidal neurons. It has been established previously (Faber and Korn 1991; Alger et al. 1996) that when $\mathrm{CV}^{2}$ changes under certain test conditions, the origin of observed phenomenon is presynaptic and mimics the alterations in neurotransmitter release. Our analyses also support this notion (Fig. 8) and we therefore conclude that the presynaptic site is involved in DSE in pyramidal cells of LA.

In conclusion, to our knowledge, this is the first successful attempt to show DSE in the LA that improves our understanding of the physiological impact of the $\mathrm{CB}_{1} \mathrm{R}$ and its ligand(s) in this center. This retrograde signaling may play a functional role in synaptic plasticity and related emotional memory in the LA. This is an important observation since the density of cannabinoid receptors in the amygdala is relatively high; and even higher in a human compared to a rat (Herkenham et al. 1990). At this time, we are not aware of any other modulators and/or third messengers (other than endocannabinoids) responsible for the retrograde signaling in the LA, however, the next possible candidates could be cyclooxygenase-2 metabolites (Kim and Alger 2004; Slanina et al. 2005) or other lipid mediators.

\section{Materials and Methods}

\section{Slice preparation}

Coronal brain slices $(300 \mu \mathrm{m}$; Fig. 1A) were prepared from 3-5-wkold C57BL/6N mice (P30.7 $\pm 0.9, n=30$ ) using an extracellular physiological solution (see Electrophysiology). These mice were first anaesthetized with isoflurane before the preparation. Animal use was in accord with the procedures approved by local committee.

\section{Electrophysiology}

Whole-cell voltage and currents in neurons of the LA were recorded using the patch-clamp technique (Hamill et al. 1981). The electrode resistance ranged between 3 and $6 \mathrm{M} \Omega$ when filled with an intracellular solution. The series resistance, which consistently ranged between 10 and $20 \mathrm{M} \Omega$ was not compensated. The input resistance of recorded pyramidal neurons had a mean value of $357.4 \pm 19.9 \mathrm{M} \Omega(n=35)$. Both spontaneous and evoked excitatory postsynaptic currents (EPSCs) were recorded at a holding potential of $-70 \mathrm{mV}$. EPSCs were evoked using either a bipolar tungsten stimulation electrode or sliver painted pipettes (Kodirov et al. 2006) filled with equilibrated ACSF (see below). In order to record evoked EPSCs, the afferent fibers of the external capsule (cortical afferents) were continuously stimulated at $0.33 \mathrm{~Hz}(100-$ $150 \mu \mathrm{s}$ pulse width) and after a stable baseline period the postsynaptic neuron was depolarized to $0 \mathrm{mV}$. The intensity of stimulation was adjusted to evoke unitary EPSCs without failures. The APs were evoked in response to depolarizing current injection (50-100 pA). The pyramidal cells were identified by frequency adaptation properties of their APs (Fig. 1C). The extracellular physiological solution contained $(\mathrm{mM}) 119 \mathrm{NaCl}, 2.5 \mathrm{KCl}, 1.25$ $\mathrm{NaH}_{2} \mathrm{PO}_{4}, 1 \mathrm{MgSO}_{4}, 2.5 \mathrm{CaCl}_{2}, 26 \mathrm{NaHCO}_{3}$, and 10 glucose. This 
solution was equilibrated with $95 \% \mathrm{O}_{2}$ and $5 \% \mathrm{CO}_{2}(\mathrm{pH}=7.4)$ and was used also for the brain slice preparation. The pipette solution was composed $(\mathrm{mM})$ of $120 \mathrm{~K}^{+}$-gluconate, $5 \mathrm{NaCl}, 1 \mathrm{MgCl}_{2}$, 10 HEPES, 0.2 EGTA, $2 \mathrm{Mg}_{2} \mathrm{ATP}$, and $0.2 \mathrm{NaGTP}$ (pH 7.2 adjusted with $\mathrm{KOH})$. In order to chelate the intracellular $\mathrm{Ca}^{2+}$ the concentration of EGTA was increased to $10 \mathrm{mM}$ in $\mathrm{K}^{+}$-gluconate-based pipette solution. In some experiments we used the equimolar concentrations of either $\mathrm{CsCl}$ or Cs-methane-sulfonate instead of $\mathrm{K}^{+}$-gluconate. All experiments were performed at room temperature $\left(\sim 24^{\circ} \mathrm{C}\right)$.

\section{Data acquisition and analysis}

The pClamp, Mini Analysis, Origin, and Microsoft Excel software were used for data acquisition and subsequent analyses. The current signals were filtered at $1 \mathrm{kHz}$ and the sampling interval was $2.5-5 \mathrm{kHz}$. For the cumulative sEPSCs' graphs, the frequency (Figs. 2, 3: bin size $10 \mathrm{~ms}$ ) and amplitude (2 pA equal bins) of synaptic events were collected under baseline conditions and immediately after a $10 \mathrm{~s}$ depolarization in each individual experiment. The data are presented as the mean \pm SEM and $n$ corresponds to the number of experiments. Paired student's $t$-test (two-tailed) was used for data group comparison; cumulative probability curves were compared with the Kolmogorov-Smirnov test; $P<0.05$ was considered significant.

\section{Acknowledgments}

The support of the Deutsche Forschungsgemeinschaft (SFB-TRR58 Fear, Anxiety, Anxiety Disorders) is gratefully acknowledged. We greatly appreciate the excellent technical support of Martin Purrio and the critical reading of the manuscript by Jennifer Falk, Marcos Cantu, and Jennifer Bagley. Finally, the helpful efforts of two anonymous reviewers are appreciated.

\section{References}

Alger BE, Pitler TA, Wagner JJ, Martin LA, Morishita W, Kirov SA, Lenz RA. 1996. Retrograde signalling in depolarization-induced suppression of inhibition in rat hippocampal CA1 cells. J Physiol 496: 197-209.

Ali AB. 2007. Presynaptic inhibition of $\mathrm{GABA}_{\mathrm{A}}$ receptor-mediated unitary IPSPs by cannabinoid receptors at synapses between CCK-positive interneurons in rat hippocampus. J Neurophysiol 98: 861-869.

Azad SC, Eder M, Marsicano G, Lutz B, Zieglgansberger W, Rammes G. 2003. Activation of the cannabinoid receptor type 1 decreases glutamatergic and GABAergic synaptic transmission in the lateral amygdala of the mouse. Learn Mem 10: 116-128.

Brown SP, Brenowitz SD, Regehr WG. 2003. Brief presynaptic bursts evoke synapse-specific retrograde inhibition mediated by endogenous cannabinoids. Nat Neurosci 6: 1048-1057.

Chen K, Ratzliff A, Hilgenberg L, Gulyas A, Freund TF, Smith M, Dinh TP, Piomelli D, Mackie K, Soltesz I. 2003. Long-term plasticity of endocannabinoid signaling induced by developmental febrile seizures. Neuron 39: 599-611.

Chevaleyre V, Heifets BD, Kaeser PS, Sudhof TC, Castillo PE. 2007. Endocannabinoid-mediated long-term plasticity requires cAMP/PKA signaling and RIM1 $\alpha$. Neuron 54: 801-812.

Devane W, Axelrod J. 1994. Enzymatic synthesis of anandamide, an endogenous ligand for the cannabinoid receptor, by brain membranes. Proc Natl Acad Sci 91: 6698-6701.

Diana MA, Marty A. 2004. Endocannabinoid-mediated short-term synaptic plasticity: Depolarization-induced suppression of inhibition (DSI) and depolarization-induced suppression of excitation (DSE). Br J Pharmacol 142: 9-19.

Duguid IC, Smart TG. 2004. Retrograde activation of presynaptic NMDA receptors enhances GABA release at cerebellar interneuron-Purkinje cel synapses. Nat Neurosci 7: 525-533.

Faber DS, Korn H. 1991. Applicability of the coefficient of variation method for analyzing synaptic plasticity. Biophys J 60: 1288-1294.

Fortin DA, Levine ES. 2007. Differential effects of endocannabinoids on glutamatergic and GABAergic inputs to layer 5 pyramidal neurons. Cereb Cortex 17: 163-174.

Gallagher M, Kapp BS, Musty RE, Driscoll PA. 1977. Memory formation: Evidence for a specific neurochemical system in the amygdala. Science 198: $423-425$.

Gewirtz JC, Davis M. 1997. Second-order fear conditioning prevented by blocking NMDA receptors in amygdala. Nature 388: 471-474.
Hamill OP, Marty A, Neher E, Sakmann B, Sigworth FJ. 1981. Improved patch-clamp techniques for high-resolution current recording from cells and cell-free membrane patches. Pflugers Arch 391: 85-100.

Hashimotodani Y, Ohno-Shosaku T, Kano M. 2007. Presynaptic monoacylglycerol lipase activity determines basal endocannabinoid tone and terminates retrograde endocannabinoid signaling in the hippocampus. J Neurosci 27: 1211-1219.

Herkenham M, Lynn A, Little M, Johnson M, Melvin L, de Costa B, Rice K. 1990. Cannabinoid receptor localization in brain. Proc Natl Acad Sci 87: 1932-1936.

Hohmann AG, Suplita RL, Bolton NM, Neely MH, Fegley D, Mangieri R, Krey JF, Michael Walker J, Holmes PV, Crystal JD, et al. 2005. An endocannabinoid mechanism for stress-induced analgesia. Nature 435 : 1108-1112.

Katona I, Rancz EA, Acsady L, Ledent C, Mackie K, Hajos N, Freund TF. 2001. Distribution of CB1 cannabinoid receptors in the amygdala and their role in the control of GABAergic transmission. J Neurosci 21: 9506-9518.

Kim J, Alger BE. 2004. Inhibition of cyclooxygenase- 2 potentiates retrograde endocannabinoid effects in hippocampus. Nat Neurosci 7: 697-698.

Kodirov SA, Takizawa S, Joseph J, Kandel ER, Shumyatsky GP, Bolshakov VY. 2006. Synaptically released zinc gates long-term potentiation in fear conditioning pathways. Proc Natl Acad Sci 103: 15218-15223.

Kreitzer AC, Regehr WG. 2001. Retrograde inhibition of presynaptic calcium influx by endogenous cannabinoids at excitatory synapses onto Purkinje cells. Neuron 29: 717-727.

Mahanty NK, Sah P. 1998. Calcium-permeable AMPA receptors mediate long-term potentiation in interneurons in the amygdala. Nature 394: 683-687.

Marsicano G, Wotjak CT, Azad SC, Bisogno T, Rammes G, Cascio MG, Hermann H, Tang J, Hofmann C, Zieglgansberger W, et al. 2002. The endogenous cannabinoid system controls extinction of aversive memories. Nature 418: 530-534.

McKernan MG, Shinnick-Gallagher P. 1997. Fear conditioning induces a lasting potentiation of synaptic currents in vitro. Nature 390: 607611.

Morgane PJ, Kosman AJ. 1957. Alterations in feline behaviour following bilateral amygdalectomy. Nature 180: 598-600.

Nader K, Schafe GE, Le Doux JE. 2000. Fear memories require protein synthesis in the amygdala for reconsolidation after retrieval. Nature 406: $722-726$.

Pavlov IP. 1927. Conditioned reflexes. Dover, New York.

Pitler TA, Alger BE. 1994. Depolarization-induced suppression of GABAergic inhibition in rat hippocampal pyramidal cells: $G$ protein involvement in a presynaptic mechanism. Neuron 13: 1447-1455.

Royer S, Pare D. 2003. Conservation of total synaptic weight through balanced synaptic depression and potentiation. Nature 422: 518-522.

Slanina KA, Roberto M, Schweitzer P. 2005. Endocannabinoids restrict hippocampal long-term potentiation via CB1. Neuropharmacology 49: 660-668.

Stella N, Schweitzer P, Piomelli D. 1997. A second endogenous cannabinoid that modulates long-term potentiation. Nature 388: 773-778.

Straiker A, Mackie K. 2005. Depolarization-induced suppression of excitation in murine autaptic hippocampal neurones. J Physiol 569: 501-517.

Takahashi KA, Linden DJ. 2000. Cannabinoid receptor modulation of synapses received by cerebellar Purkinje cells. J Neurophysiol 83: 11671180.

Tzounopoulos T, Rubio ME, Keen JE, Trussell LO. 2007. Coactivation of preand postsynaptic signaling mechanisms determines cell-specific spiketiming-dependent plasticity. Neuron 54: 291-301.

Uchigashima M, Narushima M, Fukaya M, Katona I, Kano M, Watanabe M. 2007. Subcellular arrangement of molecules for 2-arachidonoylglycerol-mediated retrograde signaling and its physiological contribution to synaptic modulation in the striatum. J Neurosci 27: 3663-3676.

Vincent P, Armstrong CM, Marty A. 1992. Inhibitory synaptic currents in rat cerebellar Purkinje cells: Modulation by postsynaptic depolarization. J Physiol 456: 453-471.

Williams JH, Errington ML, Lynch MA, Bliss TV. 1989. Arachidonic acid induces a long-term activity-dependent enhancement of synaptic transmission in the hippocampus. Nature 341: 739-742.

Wilson RI, Kunos G, Nicoll RA. 2001. Presynaptic specificity of endocannabinoid signaling in the hippocampus. Neuron 31: 453462.

Zhu PJ, Lovinger DM. 2005. Retrograde endocannabinoid signaling in a postsynaptic neuron/synaptic bouton preparation from basolateral amygdala. J Neurosci 25: 6199-6207.

Received October 8, 2009; accepted in revised form November 12, 2009 


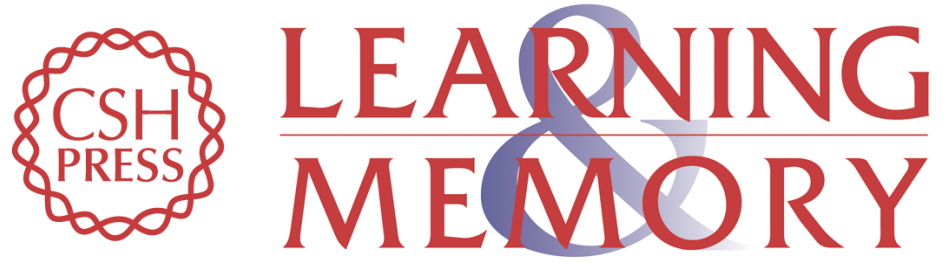

\section{Endogenous cannabinoids trigger the depolarization-induced suppression of excitation in the lateral amygdala}

Sodikdjon A. Kodirov, Julia Jasiewicz, Parisa Amirmahani, et al.

Learn. Mem. 2010, 17:

Access the most recent version at doi:10.1101//m.1663410

References This article cites 38 articles, 9 of which can be accessed free at: http://learnmem.cshlp.org/content/17/1/43.full.html\#ref-list-1

License

Email Alerting Receive free email alerts when new articles cite this article - sign up in the box at the Service top right corner of the article or click here. 